\title{
A NOTE ON THE RATES OF CONVERGENCE OF DOUBLE SEQUENCES
}

\author{
METIN BAŞARIR
}

\begin{abstract}
In this paper, we define the rates of convergence of double sequences and give some theorems on the rates of convergence of bounded double null sequences with real terms.
\end{abstract}

\section{INTRODUCTION}

The rates of convergence of single sequences have been studied in [2], [3], [4], [5], [7,p.298-301], [8], [9] and [10]. M.Bajraktarevic [8],[9] gave some theorems on rates of convergence of single null sequences. The aim of this short paper is to extend the concept of rate of convergence of sequences into double sequences and is to give the analogues of fundamental theorems appeared in [8].

First, we give the definition of convergence of double sequences. The original Pringsheim's definition ([5], Vol.II, p.303) says that a sequence $\left\{x_{m n}\right\}_{m, n=0}^{\infty}$ converges to the limit $t$ if for every $\varepsilon>0$ there is an $N$ such that

$$
\left|x_{m n}-t\right|<\varepsilon \text { whenever } \min (m, n)>N .
$$

A double sequence $x$ is bounded if

$$
\|x\|=\sup _{i, j \geq 0}\left|x_{i j}\right|<\infty .
$$

We notice that a convergent double sequence need not be bounded, as the example in [6, p.321].

Definition 1. Suppose that $x=\left\{x_{m n}\right\}_{m, n=0}^{\infty}$ and $y=\left\{y_{m n}\right\}_{m, n=0}^{\infty}$ are two bounded double convergent sequences of real terms and that $\lim _{m, n \rightarrow \infty} x_{m n}$ $=X, \lim _{m, n \rightarrow \infty} y_{m n}=Y, y_{m n} \neq Y$ for all $m, n$. We say that $x$ converges faster, slower, at the same rate or completely incomparable rates as $y$ if,

$$
\lim _{m, n \rightarrow \infty} \frac{x_{m n}-X}{y_{m n}-Y}=0
$$

2010 Mathematics Subject Classification. 40A05. 


$$
\begin{gathered}
\lim _{m, n \rightarrow \infty} \frac{x_{m n}-X}{y_{m n}-Y}=+\infty \\
0<\lim _{\frac{m, n \rightarrow \infty}{\lim _{m n}}\left|\frac{x_{m n}-X}{y_{m n}}\right| \leq \lim _{m, n \rightarrow \infty}\left|\frac{x_{m n}-X}{y_{m n}-Y}\right|<+\infty}\left|\frac{x_{m n}-X}{y_{m n}-Y}\right|=0 \text { and } \\
\lim _{m, n \rightarrow \infty}\left|\frac{x_{m n}-X}{y_{m n}-Y}\right|=+\infty
\end{gathered}
$$

hold, respectively, where $\lim _{m, n \rightarrow \infty}=\liminf _{m n}$ and $\varlimsup_{\lim _{m, n \rightarrow \infty}}=\lim \sup _{m n}$. Term by term quotients are considered in this definition.

Throughout the paper, $A$ will denote a collection of bounded double sequences, each of which converges to zero and such that each term is different from zero.

\section{MAin Results}

We have

Theorem 1. There exists a bounded double positive null sequence $z=$ $\left\{z_{m n}\right\}_{m, n=0}^{\infty}$, all of whose terms are non-zero, which converges faster than each $x \in A$ if and only if there exists a collection $\left\{A_{n}\right\}_{n=1}^{\infty}$ of sub-collections of $A$ with the following properties.

$$
A_{n} \subseteq A_{n+1} \text { for every natural number } n, \cup_{n=1}^{\infty} A_{n}=A
$$

and

$$
\begin{aligned}
& y_{k l}=\inf _{x \in A_{p}}\left(\left|x_{k l}\right|\right)>0 \text { for every } \min (k, l) \\
& \quad \geq p \geq n_{0} \text {, for some fixed positive integer } n_{0} .
\end{aligned}
$$

Proof. To see that (5) and (6) are necessary conditions, suppose that $z=$ $\left\{z_{m n}\right\}_{m, n=0}^{\infty}$ is a double bounded positive null sequence, all of whose terms are different from zero, that converges faster than each $x \in A$. Let set

$$
A_{n}=\left\{x: 0<z_{k l} \leq\left|x_{k l}\right| \text {, for every } \min (k, l) \geq n\right\} .
$$

Clearly (5) holds for the collection $\left\{A_{n}\right\}_{n=1}^{\infty}$ of sub-collections of $A$ by the definition of converging faster. Furthermore, there exists a positive integer $n_{0}$ such that $A_{p} \neq \phi, p \geq n_{0}$. If $\min (k, l) \geq p \geq n_{0}, y_{k l}=\inf _{x \in A_{p}}\left(\left|x_{k l}\right|\right) \geq$ $z_{k l}>0$ so that (6) is satisfied.

To see the sufficiency of the conditions, suppose that $\left\{A_{n}\right\}_{n=1}^{\infty}$ is a collection of sub-collections of $A$ satisfying (5) and (6). For each $x \in A$, $p_{x}=\min \left\{p: p \geq n_{0}, x \in A_{p}\right\}$. Therefore we have

$$
0<y_{k l} \leq\left|x_{k l}\right| \text { for every } \min (k, l) \geq p_{x} .
$$


Then $y_{k l} \rightarrow 0$, because of $x_{k l} \rightarrow 0$. Let $z=\left\{z_{k l}\right\}_{k, l=0}^{\infty}$, where $z_{k l}=y_{k l}^{2}$ for every $k, l$. Then $z=\left\{z_{k l}\right\}$ is a bounded double positive null sequence and

$$
0<\frac{z_{k l}}{\left|x_{k l}\right|}=y_{k l}\left(\frac{y_{k l}}{\left|x_{k l}\right|}\right) \leq y_{k l}, \min (k, l) \geq p_{x} .
$$

and $\frac{z_{k l}}{\left|x_{k l}\right|} \rightarrow 0$. So that $z$ converges faster than $x$, for every $x$.

Theorem 2. There exists a double bounded positive null sequence $z=$ $\left\{z_{m n}\right\}_{m, n=0}^{\infty}$ all of whose terms are non-zero, which converges slower than each $x \in A$ if and only if there exists a collection $\left\{A_{n}\right\}_{n=1}^{\infty}$ non-empty subcollections of $A$, a strictly increasing sequence $\left\{N_{n}\right\}_{n=1}^{\infty}$ of positive integers and a decreasing null sequence $\left\{\varepsilon_{n}\right\}_{n=1}^{\infty}$ with $0<\varepsilon_{n+1}<\varepsilon_{n}<1$ for all $n \geq 1$ such that

$$
\begin{gathered}
A_{n} \subseteq A_{n+1} \text { for every } n, \cup_{n=1}^{\infty} A_{n}=A \\
\sup _{\min (k, l) \in\left[N_{n}, N_{n+1}\right) \cap \mathbb{N}}\left\{\sup _{x \in A_{n}}\left|x_{k l}\right|\right\} \\
\leq \varepsilon_{n}, \text { for every } n \geq n_{0}, \text { for some positive integer } n_{0},
\end{gathered}
$$

where $\mathbb{N}$ is the set of positive integers.

Proof. To see that (8) and (9) are sufficient conditions, let define $y=$ $\left\{y_{m n}\right\}_{m, n=0}^{\infty}$ as follows.

$$
\begin{aligned}
& y_{i, j}= \\
& \left\{\begin{array}{cc}
\sup _{\in\left[N_{n}, N_{n+1}\right) \cap \mathbb{N}}\left\{\sup _{x \in A_{n}}\left|x_{k l}\right|\right\} \leq \varepsilon_{n}, & \text { if } \min (i, j) \in\left[N_{n}, N_{n+1}\right) \cap \mathbb{N}, n \geq n_{0} \\
1, & \text { otherwise. }
\end{array}\right.
\end{aligned}
$$

Then $y$ is a bounded double null sequence, all of whose terms are greater than zero. $z=\left\{z_{m n}\right\}_{m, n=0}^{\infty}$, where $z_{m n}=\sqrt{y_{m n}}$ for every $m, n$. Suppose $x \in A$ then $x \in A_{n}$ for every $n \geq n_{0}$, where $n_{0}$ is some fixed positive integer. If $\min (i, j) \in\left[N_{n}, N_{n+1}\right) \cap \mathbb{N}$, where $n \geq n_{0}$, then

$$
\frac{z_{i j}}{\left|x_{i j}\right|}=\frac{1}{\sqrt{y_{i j}}}\left(\frac{y_{i j}}{\left|x_{i j}\right|}\right) \geq \frac{1}{\sqrt{y_{i j}}} \geq \frac{1}{\sqrt{\varepsilon_{n}}}
$$

and therefore $z$ converges slower than each $x$.

To see that (8) and (9) are necessary conditions, suppose $z=\left\{z_{m n}\right\}$ is a bounded double positive null sequence, all of whose terms are non-zero, which converges slower than each $x \in A$. Then

$$
\lim _{m, n \rightarrow \infty} \frac{z_{m n}}{\left|x_{m n}\right|}=+\infty \text { for each } x \in A \text {. }
$$


Let

$$
A_{n}=\left\{x \in A: 0<\left|x_{k l}\right| \leq z_{k l}, \text { for all } \min (k, l) \geq n\right\} .
$$

Then clearly (8) holds and furthermore there exists a positive integer $n_{0}$ such that $A_{n} \neq \phi$, for all $n \geq n_{0}$. If $\min (k, l) \geq n \geq n_{0}$ then

$$
\sup _{x \in A_{n}}\left\{\left|x_{k l}\right|\right\} \leq z_{k l} \text {. }
$$

For all $n \geq n_{0}$, there exists a positive $N_{n}$ with $N_{n}<N_{n+1}$ such that

$$
z_{k l} \leq \varepsilon_{n}, \text { if } \min (k, l) \geq N_{n}
$$

Thus

$$
\sup _{x \in A_{n}}\left\{\left|x_{k l}\right|\right\} \leq z_{k l} \leq \varepsilon_{n}, \quad \min (k, l) \geq N_{n}, n \geq n_{0} .
$$

Then

$$
\sup _{x \in A_{n}}\left\{\left|x_{k l}\right|\right\} \leq z_{k l} \leq \varepsilon_{n}, \quad \min (k, l) \in\left[N_{n}, N_{n+1}\right) \cap \mathbb{N}, n \geq n_{0},
$$

so that (9) is satisfied.

Theorem 3. There exists a bounded double null sequence $z$, all of whose terms are non-zero such that $x$ and $z$ converge at the same rate for every $x \in A$ if and only if $x, y \in A$ implies that $x$ and $y$ converge at the same rate.

Proof. Necessity. Suppose that $z=\left\{z_{m n}\right\}$ converges at the same rate with the rate of convergence of each $x \in A$ and $z_{m n} \rightarrow 0$. Chose $x, y \in A(x \neq y)$ arbitrarily. Then

$$
\begin{aligned}
& 0<k_{1}=\lim _{m, n \rightarrow \infty}\left|\frac{z_{m n}}{x_{m n}}\right| \leq \lim _{m, n \rightarrow \infty}\left|\frac{z_{m n}}{x_{m n}}\right|=K_{1}<+\infty \\
& 0<k_{2}=\lim _{\underline{m, n \rightarrow \infty}}\left|\frac{z_{m n}}{y_{m n}}\right| \leq \lim _{m, n \rightarrow \infty}\left|\frac{z_{m n}}{y_{m n}}\right|=K_{2}<+\infty .
\end{aligned}
$$

Therefore there exists $k^{\star}$ and $K^{\star}$ numbers $\left(0<k^{\star} \leq K^{\star}<+\infty\right)$ such that for all $n$ and $m$,

$$
k^{\star} \leq\left|\frac{z_{m n}}{x_{m n}}\right| \leq K^{\star}, k^{\star} \leq\left|\frac{z_{m n}}{y_{m n}}\right| \leq K^{\star}
$$

Hence

So that

$$
\frac{k^{\star}}{K^{\star}} \leq\left|\frac{y_{m n}}{x_{m n}}\right| \leq \frac{K^{\star}}{k^{\star}} .
$$

$$
0<\lim _{\underline{m, n \rightarrow \infty}}\left|\frac{y_{m n}}{x_{m n}}\right| \leq \lim _{m, n \rightarrow \infty}\left|\frac{y_{m n}}{x_{m n}}\right|<+\infty .
$$

Sufficiency. Clearly, if we take $z \in A$ then the conclusion follows. 
Theorem 4. Suppose that $K_{1}=\left(k_{n}^{1}\right)$ and $K_{2}=\left(k_{n}^{2}\right)$ be two interwined sequences of positive integers, $\left\{A_{n}\right\}_{n=1}^{\infty}$ and $\left\{B_{n}\right\}_{n=1}^{\infty}$ be two sequences of non-empty sub-collections of $A$ and a strictly monotone sequence $N_{n}$ with the following properties.

$$
\begin{gathered}
A_{n} \subseteq A_{n+1}\left(n \in K_{1}\right), \cup_{n \in K_{1}} A_{n}=A \\
B_{n} \subseteq B_{n+1}\left(n \in K_{2}\right), \cup_{n \in K_{2}} B_{n}=A \\
y_{k l}=\inf _{x \in A_{p}}\left(\left|x_{k l}\right|\right)>0 \quad\left(\min (k, l) \geq p \geq n, n \in K_{1}\right) \\
\sup _{\min (k, l) \in\left[N_{n}, N_{n+1}\right) \cap K_{2}}\left\{\sup _{x \in B_{n}}\left|x_{k l}\right|\right\} \leq \frac{1}{n}, \quad\left(n \in K_{2}\right) .
\end{gathered}
$$

Then there exists a sequence $z=\left\{z_{m n}\right\}$ belonging to $A$ which converges to zero at a rate completely incomparable with the rate of convergence of each $x \in A$.

Proof. If $x \in A$, let $n_{x}=\min \left\{n: n \in K_{1}, x \in A_{n}\right\}$. From (12), $0<y_{k l} \leq$ $\left|x_{k l}\right|$, for all $\min (k, l) \geq n \geq n_{x},\left(n \in K_{1}\right)$. Because of $x_{m n} \rightarrow 0, y_{m n} \rightarrow 0$ $\left(m, n \in K_{1}\right)$. We define $z=\left\{z_{m n}\right\}$ such that $z_{m n}=y_{m n}^{2}\left(m, n \in K_{1}\right)$ and $\frac{z_{m n}}{\left|x_{m n}\right|} \rightarrow 0\left(m, n \rightarrow \infty, m, n \in K_{1}\right)$. Let define

$$
y_{i, j}=\sup _{\substack{\min (k, l) \\ \in\left[N_{n}, N_{n+1}\right) \cap K_{2}}}\left\{\sup _{x \in B_{n}}\left|x_{k l}\right|\right\} \leq \frac{1}{n},\left(n \in K_{2}, \min (i, j) \in\left[N_{n}, N_{n+1}\right) \cap K_{2}\right) .
$$

Then

$$
0<\left|x_{k l}\right| \leq y_{i j} \leq \frac{1}{n},\left(x \in B_{n}, \min (i, j) \in\left[N_{n}, N_{n+1}\right) \cap K_{2}, n \in K_{2}\right) .
$$

and

$$
\begin{aligned}
y_{k l} \rightarrow 0(k, l \rightarrow \infty), & \frac{y_{k l}}{\left|x_{k l}\right|} \geq 1, \\
& \left(x \in B_{n}, \min (k, l) \in\left[N_{n}, N_{n+1}\right) \cap K_{2}, n \in K_{2}\right) .
\end{aligned}
$$

Suppose $z_{k l}=\sqrt{y_{k l}},\left(\min (k, l) \in\left[N_{n}, N_{n+1}\right) \cap K_{2}, n \in K_{2}\right)$ and for others $z_{k l}$ it can take arbitrary numbers with $z_{k l} \rightarrow 0$. Let $x \in A$. Then, for all $n \in K_{2}, x \in B_{n}$ and for all $\min (k, l) \in\left[N_{n}, N_{n+1}\right) \cap K_{2}$, we have

Hence

$$
\frac{z_{k l}}{\left|x_{k l}\right|}=\frac{1}{\sqrt{y_{k l}}}\left(\frac{y_{k l}}{\left|x_{k l}\right|}\right) \geq \frac{1}{\sqrt{y_{k l}}} \rightarrow \infty \quad(k, l \rightarrow \infty) .
$$

$$
\lim _{m, n \rightarrow \infty} \frac{z_{m n}}{\left|x_{m n}\right|}=0, \overline{\lim _{m, n \rightarrow \infty}} \frac{z_{m n}}{\left|x_{m n}\right|}=+\infty \text { for every } x \in A .
$$

If we take $A_{n}=B_{n}\left(n \in K_{2}\right)$ in Theorem 4, we have the following theorem. 
Theorem 5. Suppose that $A$ is a collection of bounded double sequences, each of which converges to zero and such that each term is different from zero. Then for there to exists a sequence $z=\left\{z_{m n}\right\}_{m, n=0}^{\infty}, z_{m n} \neq 0$ for every $m$ and $n$, which converges to zero at a rate completely incomparable with the rate of convergence of each $x \in A$, it is sufficient for there to exists two interwining sequences $K_{1}$ and $K_{2}$ of positive integers, a sequence $\left\{A_{n}\right\}_{n=1}^{\infty}$ of non-empty sub-collections of $A$ and a strictly monotone sequence $\left\{N_{n}\right\} \subset K_{2}$ with the following properties.

$$
\begin{gathered}
A_{n} \subseteq A_{n+1} \text { for all } n \text { and } \cup_{n \in K_{1}} A_{n}=A \\
y_{k l}=\inf _{x \in A_{p}}\left(\left|x_{k l}\right|\right)>0 \quad\left(\min (k, l) \geq n, n \in K_{1}\right) \\
\sup _{\min (k, l) \in\left[N_{n}, N_{n+1}\right) \cap K_{2}}\left\{\sup _{x \in A_{n}}\left|x_{k l}\right|\right\} \leq \frac{1}{n}, \quad\left(n \in K_{2}\right) .
\end{gathered}
$$

\section{REFERENCES}

[1] A. Zygmund, Trigonometric Series, University Press, (Cambridge, 1959).

[2] D. F. Dawson, Some rate invariant sequence transformations, Proc. Amer. Math. Soc., 15 (5) (1964), 710-714.

[3] H. I. Miller, A class of non-rate invariant transformations, Rad. Odjeljenje Prir. Mat. Nauka, XLV/12 (1973), 41-43.

[4] H. I. Miller, Rates of convergence and summability, Rad. Odjeljenje Prir. Mat. Nauka, XLV/12 (1973), 85-92.

[5] H. I. Miller, A note on matrix summability and rates of convergence, Mat. Vesnik, 10 (25) (1973), 145-147.

[6] I. Szalay, On the strong Cesaro summability of double series, Anal. Math., 15 (1989), 321-341.

[7] K. Knopp, Theory and Applications of Infinite Series, Berlin, 1931.

[8] M. Bajraktarevic, Sur quelques problemes concernant la vitesse de convergence at la sommabilite des suites, Rad. Odjeljenje Prir. Mat. Nauka, LII/14 (1974), 5-27.

[9] M. Bajraktarevic, Sur quelques problèmes concernant la vitesse de convergence at la sommabilité des suites (II), Publ. Inst. Math. (Beograd), (N.S.), 16 (30) (1973), 25-30.

[10] M. Başarır, On rates of convergence of sequences, J. Orissa Math. Soc., 7 (2) (1988), 89-98.

(Received: October 11, 2013)

Department of Mathematics

Sakarya University

54187, Sakarya

Turkey

basarir@sakarya.edu.tr 